\title{
Resistance to second-line injectables and treatment outcomes in multidrug-resistant and extensively drug-resistant tuberculosis
}

\section{cases}

\author{
G.B. Migliori*, C. Lange ${ }^{\#}$, R. Centis*, G. Sotgiu“, R. Mütterlein ${ }^{+}$, H. Hoffmann ${ }^{\S}$,

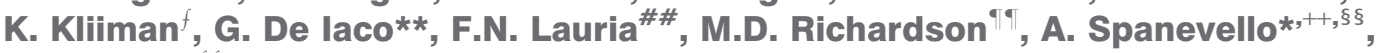 \\ D.M. Cirillo ${ }^{f f}$ and TBNET Study Group ${ }^{\star \star *}$
}

ABSTRACT: No information is currently available on the influence of injectable second-line drugs on treatment outcomes of multidrug-resistant (MDR) and extensively drug-resistant (XDR) tuberculosis (TB) patients.

To investigate this issue, a large series of MDR- and XDR-TB cases diagnosed in Estonia, Germany, Italy and the Russian Federation (Archangels Oblast) between 1999 and 2006 were analysed. All study sites performed drug susceptibility testing for first- and second-line anti-TB drugs, laboratory quality assurance and treatment delivery according to World Health Organization recommendations.

Out of 4,583 culture-confirmed cases, 240 MDR- and 48 XDR-TB cases had a definitive outcome recorded (treatment success, death, failure). Among MDR- and XDR-TB cases, capreomycin resistance yielded a higher proportion of failure and death than capreomycin-susceptible cases. Resistance to capreomycin was independently associated with unfavourable outcome (logistic regression analysis: odds ratio 3.51 ).

In the treatment of patients with multidrug-resistant and extensively drug-resistant tuberculosis, resistance to the injectable drug capreomycin was an independent predictor for therapy failure in this cohort. As Mycobacterium tuberculosis drug resistance is increasing worldwide, there is an urgent need for novel interventions in the fight against tuberculosis.

KEYWORDS: Drug resistance, extensively drug-resistant tuberculosis, injectable second-line drugs, multidrug-resistant tuberculosis, tuberculosis

xtensively drug-resistant (XDR) tuberculosis (TB) is defined as resistance to at least rifampicin and isoniazid, plus resistance to any fluoroquinolone and at least one of three injectable anti-TB drugs (capreomycin, kanamycin, or amikacin) [1-4]. Multidrugresistant (MDR)-TB is defined as resistance to at least rifampicin and isoniazid. Preliminary reports from a number of sources following the XDR-TB outbreak in the town of Tugela Ferry, South Africa, suggested that XDR-TB may be almost incurable [4-6]. The XDR-TB-defining drugs are those considered essential to achieve successful outcomes in MDR-TB cases [1-4]. As XDR-TB is still a newly described disease, evidence on the role of the different classes of anti-TB drugs in its treatment is scant and slowly accumulating.
After demonstrating that XDR-TB patients have a higher risk of death and failure than those with MDR-TB [4, 7, 8], two TuBerculosis Network (TBNET) studies $[9,10]$ showed that: 1) resistance to additional first-line drugs (other than isoniazid and rifampicin) is a predictor of adverse outcomes [9]; and 2) resistance to fluoroquinolones contributes to increased risk of death and failure in these cases [10]. However, no information is presently available on the influence of injectable second-line drugs on treatment outcomes among MDR- or XDR-TB cases.

\section{METHODS}

Drug resistance and treatment outcome data were reviewed for all 4,583 culture-confirmed TB cases diagnosed consecutively by the TB clinical reference centres between 2003 and 2006
AFFILIATIONS

*WHO Collaborating Centre for TB and Lung Diseases, Fondazione S. Maugeri, Care and Research Institute, Tradate,

"Hygiene and Preventive Medicine Institute, University of Sassari, Sassari, ${ }^{\star *}$ E. Morelli Hospital, Reference Hospital for MDR and HIV TB, Sondalo, \#\# National Institute for Infectious Diseases, L. Spallanzani, Rome, ${ }^{+}$Fondazione S. Maugeri, Care and Research Institute, Cassano delle Murge ${ }^{\S \S}$ University of Foggia, Foggia, and ${ }^{f f}$ Emerging Bacterial Pathogens Unit, S. Raffaele Scientific Institute, Milano, Italy. \#Division of Clinical Infectious Diseases, Medical Clinic, Research Centre Borstel, Borstel,

+Dept of Medicine, Parsberg Hospital, Parsberg, and

§Supranational Reference Laboratory of Tuberculosis, IML Asklepios Clinic Munich-Gauting, Munich-Gauting, Germany.

f University of Tartu, Tartu, Estonia.

"TB Program, PATH, Seattle, WA, USA ${ }^{\star \star *}$ For a full list of the members of the TBNET Study Group, please refer to the Acknowledgements section.

\section{CORRESPONDENCE}

G.B. Migliori, WHO Collaborating Centre for TB and Lung Diseases, Fondazione S. Maugeri, Care and Research Institute, via Roncaccio 16, 21049, Tradate, Italy. Fax: 39331829402

E-mail: giovannibattista.migliori@fsm.it

Received:

February 252008

Accepted after revision:

March 142008

STATEMENT OF INTEREST

None declared.

European Respiratory Journal Print ISSN 0903-1936 Online ISSN 1399-3003 


\begin{tabular}{|c|c|c|c|c|c|c|}
\hline TABLE 1 & $\begin{array}{l}\text { Outcomes of multidrug-resistan } \\
\text { resistant and susceptible to inje } \\
\text { (Archangels Oblast) }^{\#}\end{array}$ & $\begin{array}{l}\text { rculosis }(\mathrm{ML} \\
\text { second-lin }\end{array}$ & $\begin{array}{l}\text { and exte } \\
\text { gs in Esto }\end{array}$ & $\begin{array}{l}\text { ly drug-resist } \\
\text { iermany, Italy }\end{array}$ & $\begin{array}{l}\text { berculosis ( } \\
\text { he Russian }\end{array}$ & $\begin{array}{l}\text { TB) cases } \\
\text { ration }\end{array}$ \\
\hline Outcome & Capreomycin & Kanamycin & Amikacin & Capreomycin & Kanamycin & Amikacin \\
\hline Resistant & 9 (39) & $72(66)$ & $15(72)$ & $4(36)$ & $22(48)$ & $8(36)$ \\
\hline Susceptible & $156(72)$ & $93(71)$ & $150(69)$ & $18(49)$ & $0(0)$ & 14 (54) \\
\hline \multicolumn{7}{|l|}{ Died } \\
\hline Resistant & $5(22)$ & $20(18)$ & $3(14)$ & $4(36)$ & $12(26)$ & $8(36)$ \\
\hline Susceptible & $38(17)$ & $23(18)$ & $40(18)$ & $10(27)$ & $2(100)$ & $6(23)$ \\
\hline \multicolumn{7}{|l|}{ Failure } \\
\hline Susceptible & $217(100)$ & $130(100)$ & $219(100)$ & 37 (100) & $2(100)$ & $26(100)$ \\
\hline
\end{tabular}

Data are presented as $n(\%)$. The percentage was calculated using the total number of cases resistant or susceptible to the given drug as a denominator. ${ }^{*}:$ includes cases resistant to one and more than one injectable.

in Germany (Borstel, Munich-Gauting, Grosshansdorf and Bad-Lippspringe) and Italy (Sondalo, Milan and Rome), between 2001 and 2004 in Estonia (Tallin and Tartu), and between 1999 and 2001 in the Russian Federation (Archangels Oblast) $[4,9,11,12]$. Standard World Health Organization (WHO) definitions for MDR-TB, XDR-TB and treatment outcome (treatment success, died, failure, default and transferred out) were used [9]. Inclusion criteria for this analysis included all 288 cases with laboratory-confirmed MDR- or XDR-TB for whom a definitive treatment end-point was recorded (treatment success, treatment failure or death). Cases were excluded if they did not meet the definition of confirmed MDR- or XDR-TB, or if they were confirmed MDRor XDR-TB cases but did not have a definitive treatment outcome recorded. Thus, MDR- and XDR-TB cases without a definitive outcome were excluded from the analysis, including $77(18 \%)$ cases still undergoing treatment, $57(13 \%)$ who defaulted and three $(0.7 \%)$ who transferred out.

An analysis was performed in order to assess potential differences between the included and excluded groups that could bias results. No significant differences were found between MDR- and XDR-TB cases included and excluded from the multivariate analysis for the main demographic variables (age, sex and immigrant status) or the number of drugs to which the Mycobacteriun tuberculosis strain was resistant.

Drug susceptibility testing (DST) for first-line anti-TB drugs (isoniazid, rifampicin, ethambutol, streptomycin and pyrazinamide) and second-line anti-TB drugs was performed according to WHO recommendations by quality-assured laboratories and re-tested at the WHO Supranational Reference Laboratories (Rome, Milan, Borstel, MunichGauting, Stockholm (Sweden) and Oslo (Norway)) [13, 14]. At all sites, regimens to treat MDR- and XDR-TB cases were tailored to the DST results according to WHO recommendations. Each of the locations had access to all categories of second-line drugs during the study period (the injectable agents amikacin, capreomycin and kanamycin; fluoroquinolones; and the oral agents ethionamide/prothionamide, para-aminosalycilic acid and cycloserine). Third-line agents (amoxicillin/clavulanic acid, clarithromycin, clofazimine) were available in all locations except Archangels Oblast.

The prevalence of resistance to injectable second-line drugs (capreomycin, kanamycin or amikacin) was analysed. Outcomes were compared using the Chi-squared test (categorical variables) or an unpaired t-test (continuous variables). Logistic regression analysis was performed. The following variables were included in the statistical analysis: country, sex, age at the time of diagnosis, immigrant status, DST results (isoniazid, rifampicin, ethambutol, pyrazinamide, streptomycin, any fluoroquinolone, amikacin, capreomycin, kanamycin), treatment outcomes and MDR- or XDR-TB status.

The study was approved by the ethical committee of the coordinating centre (WHO Collaborating Centre for TB and Lung Diseases, S. Maugeri Foundation Tradate, Italy). Patient confidentiality was ensured by circulation of only coded information to all investigators except the treating physicians.

\section{RESULTS}

Of 4,583 culture-confirmed cases (Italy: $n=2,140$; Germany: $\mathrm{n}=748$; Estonia: $\mathrm{n}=900$; Archangels Oblast: $\mathrm{n}=795), 361(7.9 \%)$ were MDR-TB and 64 (1.4\%) were XDR-TB cases. Of these, 240 (66.5\%) MDR-TB cases (Italy: $n=26$; Germany: $n=27$; Estonia: $\mathrm{n}=153$; Archangels Oblast: $\mathrm{n}=34)$ and $48(75 \%)$ XDR-TB cases (Italy: $\mathrm{n}=4$; Estonia: $\mathrm{n}=44$ ) had a definitive outcome recorded and were included in the analysis (tables 1 and 2). In the final sample of 288 cases for analysis, 124 (43\%) cases were resistant to one or more of the second-line injectables. In total, 43 (15\%) 


\begin{tabular}{|c|c|c|c|c|c|c|c|}
\hline TABLE 2 & \multicolumn{7}{|c|}{$\begin{array}{l}\text { Outcomes of extensively drug-resistant tuberculosis (XDR-TB) and multidrug-resistant tuberculosis (MDR-TB) cases } \\
\text { susceptible and resistant to one or more injectable second-line drug in Estonia, Germany, Italy and Russian Federation } \\
\text { (Archangels Oblast) }\end{array}$} \\
\hline \multicolumn{8}{|l|}{ MDR-TB } \\
\hline Susceptible & & 89 (72) & $23(18)$ & $12(10)$ & $124(100)$ & 1 & 1 \\
\hline Resistant to & 2 drugs & $8(40)$ & $4(20)$ & $8(40)$ & $20(100)$ & $1.95(1.19-3.18)$ & $1.02(0.42-2.47)$ \\
\hline Resistant to & 3 drugs & $6(67)$ & $2(22)$ & $1(11)$ & $9(100)$ & $1.08(0.67-1.75)$ & $0.92(0.56-1.51)$ \\
\hline \multicolumn{8}{|l|}{ XDR-TB $^{+}$} \\
\hline Resistant to & 1 drug & $13(52)$ & 7 (28) & $5(20)$ & $25(100)$ & & \\
\hline Resistant to & 2 drugs & $6(40)$ & $4(27)$ & $5(33)$ & $15(100)$ & & \\
\hline Resistant to & 3 drugs & $3(37.5)$ & $3(37.5)$ & $2(25)$ & $8(100)$ & & \\
\hline Resistant to & 3 drugs & $9(53)$ & $5(29)$ & $3(18)$ & $17(100)$ & $1.31(0.93-1.84)$ & $0.92(0.56-1.51)$ \\
\hline
\end{tabular}

Data are presented as $\mathrm{n}(\%)$, unless otherwise stated. OR: odds ratio; $\mathrm{Cl}$ : confidence interval. ${ }^{*}$ : number of injectables the patient is resistant to versus susceptible strains: $\because$ : number of injectables the patient is resistant to versus susceptible strains adjusted for the following variables: country, sex, immigrant status and age; ${ }^{+}$: the comparison carried out on the overall sample and on MDR-TB cases was not possible for XDR-TB cases as, by definition, no strain was susceptible to all the three injectable drugs

cases were resistant to amikacin, $34(12 \%)$ to capreomycin and $156(54 \%)$ to kanamycin. Among the cases with any resistance to second-line injectables, 52 were resistant to more than one injectable ( 35 to two compounds and 17 to all three).

Out of the 240 MDR-TB cases with a definitive outcome reported, the proportion of cases resistant to capreomycin, kanamycin and amikacin was 9.6\% $(n=23), 45.8 \%(n=110)$ and $8.7 \%(n=21)$, respectively, and among the $48 \mathrm{XDR}-\mathrm{TB}$ cases it was $22.9 \%$ $(n=11), 95.8 \%(n=46)$ and $45.8 \%(n=22)$, respectively.

The proportion of HIV-seropositive patients was low in the sample of 288 cases: 10 (4.2\%) out of 240 in MDR-TB and one $(2.1 \%)$ out of 48 in XDR-TB cases. No significant differences in outcomes were noted for HIV-infected patients.

Capreomycin-resistant MDR-TB cases yielded a higher proportion of failure and death than capreomycin-susceptible ones (14 $(60.9 \%)$ out of 23 versus $61(28.1 \%)$ out of $217 ; p=0.0013)$. Unfavourable outcomes were similar in patients susceptible versus resistant to kanamycin and amikacin: 38 (34.5\%) out of 110 versus $37(28.5 \%)$ out of $130(p=0.31)$ and six $(28.6 \%)$ out of 21 versus $69(31.5 \%)$ out of $219(\mathrm{p}=0.78)$, respectively.

Furthermore, patients who were resistant to more than one injectable drug were more likely to achieve an unfavourable outcome. In the overall sample, 37 (33\%) out of 112 cases resistant to only one injectable had an unfavourable outcome versus $29(55.7 \%)$ out of 52 cases resistant to more than one injectable drug (odds ratio (OR) 2.56, 95\% confidence interval (CI) 1.24-5.31; $\mathrm{p}=0.006$ ). In MDR-TB cases, adverse outcomes appeared in $25(28.7 \%)$ out of 87 single-injectable resistant cases versus 15 (51.7\%) out of 29 multi-injectable resistant cases (OR 2.66, 95\% CI 1.03-6.9; $\mathrm{p}=0.024)$, and for XDR-TB in 12 (48\%) out of 25 versus $14(60.8 \%)$ out of 23 cases (OR 1.69, 95\% CI $0.46-6.25 ; \mathrm{p}=0.37)$.
A logistic regression analysis was used to compare outcomes for cases susceptible versus resistant to each of the injectable agents. Resistance to capreomycin was the only independent variable significantly associated with unfavourable outcome (OR 3.51, 95\% CI 1.67-7.36; $\mathrm{p}=0.001$ ), while resistance to amikacin (OR 1.76, 95\% CI 0.91-3.39; $\mathrm{p}=0.09$ ) and kanamycin (OR 1.57, 95\% CI 0.96-2.57; p=0.07) achieved border-line significance. After adjustment for covariates (country, sex, immigrant status and age), the corresponding ORs did not significantly change. Resistance to capreomycin (OR 3.29, 95\% CI 1.41-7.69; $\mathrm{p}=0.006$ ), amikacin (OR 0.83, 95\% CI 0.36-1.9; $\mathrm{p}=0.66$ ) and kanamycin (OR 0.81,95\% CI 0.44-1.47; $\mathrm{p}=0.49$ ) were similar after adjustments. Furthermore, a comparison of outcomes between cases susceptible to all three injectables versus cases resistant to one, two and three injectable drugs was performed (table 2). In this analysis, treatment outcomes did not appear to be poorer as the number of drugs to which a case was resistant increased.

\section{DISCUSSION}

The results of the present study suggest that resistance to capreomycin, in particular, significantly increases the risk of death and treatment failure in MDR- and XDR-TB cases, while resistance to either kanamycin or amikacin alone does not appear to be as important an indicator of poor prognosis. While the study did not show that resistance to more than one injectable leads to significantly poorer outcomes, a larger sample would help to clarify whether there is indeed a tendency toward poorer outcomes in cases with additional resistance. Outcomes for XDR-TB cases already resistant to fluoroquinolones seemed to be less affected by loss of multiple second-line injectable drugs.

The strengths of the study include: large sample size, high quality of laboratory data (all XDR-TB defining drugs tested 
and DST controlled for quality) and the capacity to provide representative data on at least two study settings, Estonia and Archangels Oblast (where all diagnosed cases were included in the study). The present results might be considered robust, as the statistical adjustment for the main covariates did not change ORs significantly. Additionally limitations of the study include: 1) the inability to assess additional factors, including variability of provider treatment practices and existence of additional comorbid conditions (other than HIV) that may confound the results; and 2) in spite of the large cohort, numbers are small when stratification per individual injectable drug is performed.

At present, caution should always be used when interpreting data related to $\mathrm{XDR}-\mathrm{TB}$ cases. In fact, universally accepted standardised approaches to testing concentrations and methods which correlate clinically with disease as well as quality assurance systems, including proficiency testing for secondline drugs, do not yet exist [9]. Further information from other MDR- and XDR-TB hot spots (e.g. South Korea, China, India and Peru) is needed to confirm the findings presently reported. A comparative analysis of the role played by the XDR-TBdefining drugs and linezolid will further contribute to clarifying their relative importance in influencing treatment outcomes.

Among the XDR-TB-defining drugs, rifampicin, fluoroquinolones and aminoglycosides are also used to treat diseases other than TB [10, 15]. The key role played by the previously mentioned drugs for the success of TB treatment suggests the urgent need to sensitise the medical community on the rational use of these life-saving compounds against other infections. This may be especially important for the use of fluoroquinolones against upper and lower respiratory tract infections in countries with a high incidence of TB $[10,11]$. Until new data from large, prospective clinical studies are available, additional guidance on the use of drugs in treatment of MDR- and XDRTB should be developed based on the new evidence of the relative importance of specific drugs in improving treatment success from observational cohort studies, such as the one presented here.

In conclusion, the present study is the first to assess the contribution of second-line injectable anti-TB drugs to treatment outcomes in MDR- and XDR-TB cases. The findings suggest that in the countries surveyed, resistance to secondline injectable drugs is, in general, widespread among drugresistant cases. In the present study, the loss of capreomycin as an effective treatment agent appears to be an important predictor of poor treatment outcomes. The implications of this finding for clinical management need to be explored in more detail before any recommendations can be considered; however, the need to consult expert clinicians for all cases of MDR-TB and XDR-TB should be emphasised, as the chances of treatment success are increased with early and effective intervention.

In this context, two parallel actions are critical at the programme level: 1) strengthening existing tuberculosis control activities to prevent new cases of multidrug-resistant and extensively drug-resistant tuberculosis; and 2) improving national capacity to diagnose and treat existing drug-resistant cases effectively, in order to provide patients with the greatest opportunity for a successful outcome. At the global level, the rational use of existing compounds must be urgently promoted to preserve their utility in treating the most difficult tuberculosis cases and intensify efforts to develop novel interventions (including new drugs and vaccines) to fight tuberculosis more effectively.

\section{ACKNOWLEDGEMENTS}

The Members of the TBNET (TuBerculosis Network in Europe Trials ) Group are as follows. J. Ortmann (Bad Lippspringe Hospital, Bad Lippspringe, Germany); D. Kirsten (Grossansdorf Hospital, Grossansdorf, Germany); S. RueschGerdes (Supranational Reference Laboratory, Borstel, Germany); F. Piana (S. Raffaele Hospital, Milan, Italy); A. Gori (Milano University, Milan, Italy); L.R. Codecasa and M. Ferrarese (Villa Marelli Institute, Milan, Italy); O.S. Toungoussova (Fondazione S. Maugeri, Cassano delle Murge, Italy and Archangels University, Archangels, Russian Federation); G. Ferrara (University of Perugia, Perugia, Italy); A. Matteelli (Brescia University, Brescia, Italy); S. De Lorenzo, P. Troupioti and G. Besozzi (Sondalo Hospital, Sondalo, Italy); L. Fattorini and E. Iona (Supranational Reference Laboratory and Istituto Superiore di Sanità, Rome, Italy); A. Gualano, P. De Mori, N. Bevilacqua and E. Girardi (INMI L. Spallanzani, Rome, Italy); M. Danilovits and V. Hollo (National Tuberculosis Programme, Tartu, Estonia); A. Mariandyshev (Archangels University, Archangels, Russian Federation).

\section{REFERENCES}

1 Extensively drug-resistant tuberculosis (XDR-TB), recommendations for prevention and control. Wkly Epidemiol Rec 2006; 81: 430-432.

2 Centers for Disease Control and Prevention (CDC). Emergence of Mycobacterium tuberculosis with extensive resistance to second-line drugs-worldwide, 2000-2004. MMWR Morb Mortal Wkly Rep 2006; 55: 301-305.

3 Migliori GB, Loddenkemper R, Blasi F, Raviglione MC. 125 years after Robert Koch's discovery of the tubercle bacillus: the new XDR-TB threat. Is "science" enough to tackle the epidemic? Eur Respir J 2007; 29: 423-427.

4 Migliori GB, Ortmann J, Girardi E, et al. Extensively drugresistant tuberculosis, Italy and Germany. Emerg Infect Dis 2007; 13: 780-782.

5 Holtz TH, Cegielski JP. Origin of the term XDR-TB. Eur Respir J 2007; 30: 396.

6 Gandhi NR, Moll A, Sturm AW, et al. Extensively drugresistant tuberculosis as a cause of death in patients coinfected with tuberculosis and HIV in a rural area of South Africa. Lancet 2006; 368: 1575-1580.

7 Migliori GB, Lange C, Girardi E, et al. Extensively drugresistant tuberculosis is worse than multidrug-resistant tuberculosis: different methodology and settings, same results. Clin Infect Dis 2008; 46: 958-959.

8 Kim H-R, Hwang SS, Kim HJ, et al. Impact of extensive drug resistance on treatment outcomes in non-HIVinfected patients with multidrug-resistant tuberculosis. Clin Infect Dis 2007; 45: 1290-1295. 
9 Migliori GB, Besozzi G, Girardi E, et al. Clinical and operational value of the extensively drug-resistant tuberculosis definition. Eur Respir J 2007; 30: 623-626.

10 Migliori GB, Lange C, Girardi E, et al. Fluoroquinolones: are they essential to treat multidrug-resistant tuberculosis? Eur Respir J 2008; 31: 904-905.

11 Kliiman K, Centis R, Migliori GB, et al. Evaluation of DOTSPlus in Estonia. Int J Tuberc Lung Dis 2005; 9: Suppl. 1, S133.

12 Toungoussova OS, Nizovtseva NI, Mariandyshev AO, Caugant DA, Sandven P, Bjune G. Impact of drug-resistant Mycobacterium tuberculosis on treatment outcome of culturepositive cases of tuberculosis in the Archangel Oblast, Russia, in 1999. Eur J Clin Microbiol Infect Dis 2004; 23: 174-179.
13 Laszlo A, Rahman M, Espinal M, Raviglione $M$, the WHO/IUATLD Network of Supranational Reference Laboratories, Quality assurance programme for drug susceptibility testing of Mycobacterium tuberculosis in the WHO/IUATLD Supranational Laboratory Network: five rounds of proficiency testing 1994-1998. Int J Tuberc Lung Dis 2002; 6: 748-756.

14 Shah NS, Wright A, Bai G-H, et al. Worldwide emergence of extensively drug-resistant tuberculosis. Emerg Infect Dis 2007; 13: 380-387.

15 Yew WW, Chau CH. Utility of fluoroquinolones in multidrug-resistant tuberculosis (MDR-TB)-a balanced view? Int J Tuberc Lung Dis 2002; 6: 174-175. 